\title{
STUDY OF SPACE CHARGE COMPENSATED LEBT FOR ESS *
}

\author{
A. Lakatos, J. Pozimski, O. Meusel, A. Jakob, H. Klein \\ Institut für Angewandte Physik, Universität Frankfurt, 60054 Frankfurt, Germany
}

\begin{abstract}
To fulfil the requirements of ESS on beam transmission and emittance growth a detailed knowledge of the physics of beam formation as well as the interaction of the $\mathrm{H}^{-}$with the residual gas is substantial. Space charge compensated beam transport using solenoids for ion optics is in favour for the Low Energy Beam Transport (LEBT) between ion source and the first RFQ. Space charge compensation reduces the electrical self fields and beam radii and therefore emittance growth due to aberrations and redistribution. Transport of $\mathrm{H}^{-}$near the ion source is negatively influenced by the dipole fields required for beam extraction and $\mathrm{e}$-dumping and the high gas pressure. The destruction of the rotational symmetry together with the space charge forces causes emittance growth and particle losses within the extraction system. High residual gas pressure near the extractor together with the high cross section for stripping will influence the transmission as well as space charge compensation. Therefore a detailed knowledge of the interaction of the residual gas with the beam and the influence of the external fields on the distribution of the compensation particles is necessary to reduce particle losses and emittance growth. Preliminary experiments using positive hydrogen ions for reference already show the influence of dipole fields on beam emittance. First measurements with $\mathrm{H}$ confirm these results. Additional information on the interactions of the residual gas with the beam ions have been gained from the measurements using the momentum and energy analyser.
\end{abstract}

\section{INTRODUCTION}

The production and low energy transport of high current negative ion beams is a key issue for future high current accelerators (i. g. spallation sources like ESS [1] (generalised perveance $\mathrm{K}=0.0035$ ) and SNS [2]). The extraction and transport of these beams have intrinsic difficulties different from positive ion beams. Different extraction and ion beam transporting schemes are under discussion [3,4], each have various positive and negative aspects.

A compact caesium free [5] $\mathrm{H}^{-}$volume source delivering a low energy high perveance beam $\left(\mathrm{H}^{-}, 6.5 \mathrm{keV}, 2.3 \mathrm{~mA}\right.$, $\mathrm{K}=0.0034$ ) has been built to study the fundamental physics of beam transport $[6,7]$.

To improve the $\mathrm{H}^{-}$to e ratio magnetic filterfields (i. g.

" Work supported by BMBF under contract no. 06 OF 841 dipoles) are used [8]. These filter fields in conjunction with dipole fields for electron dumping impede exact beam extraction simulations by destruction of the rotational symmetry. This destruction on the other hand can cause together with the influence of the dipole fields on the plasma sheath emittance growth within the extraction system, this has been proven for positive ions.

\section{EXPERIMENTAL SET UP}

A schematic drawing of our source is shown in Fig. 1. The source can be divided into three parts: the plasma generator (left), the extraction system (centre) and the dumping system (right). The plasma generator is equipped with a solenoid and a filter magnet on the front end. The filter magnet, an electrical dipole magnet, is installed near the plasma electrode.

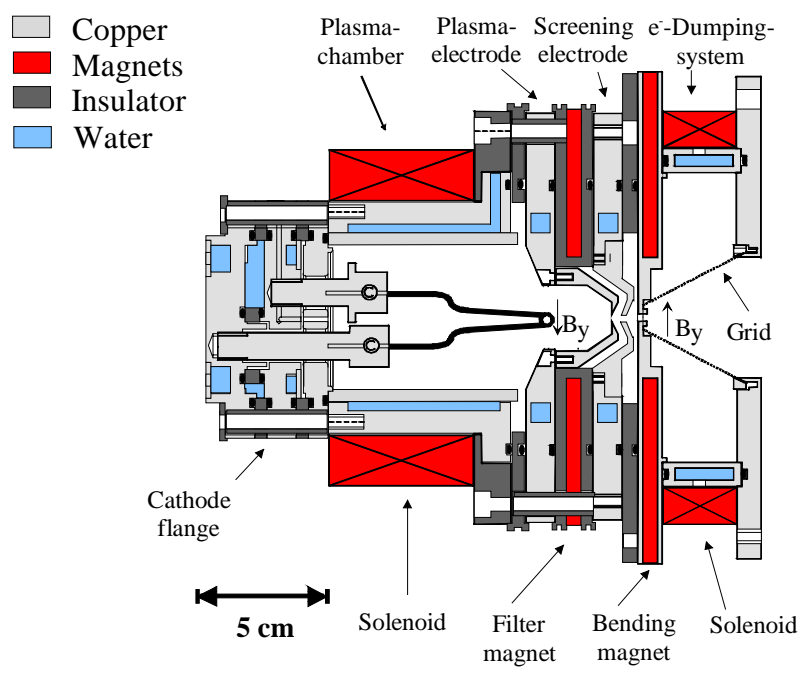

Figure 1: Schematic drawing of the ion source.

The magnetic field of the bending magnet deflects the simultaneously extracted electrons into the dumping system To reduce the magnetic field strength at the outlet aperture and therefrom the influence on beam emittance, the filter magnet and the bending magnet of the dumping system have opposite polarity. To study the fundamental behaviour of the ion source for different parameters of the plasma generator a test bench was installed. A schematic drawing of the test bench is shown in Fig. 2. Multiple beam diagnostic elements like a magnetic and electrostatic spectrometer, an emittance measurement device, a residual gas ion energy spectrometer (RGI) and Faraday cups are available along the beam path. 


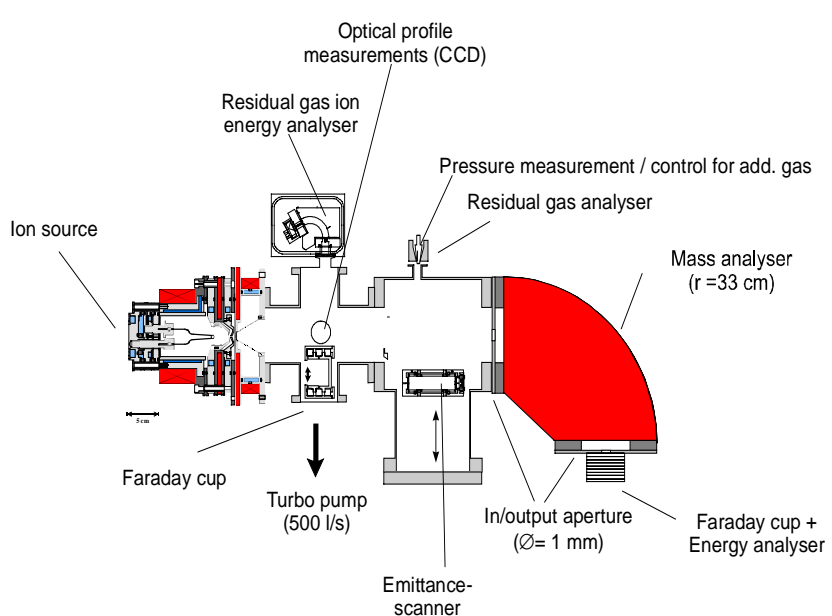

Figure 2: Schematic drawing of the experimental set up of the test bench for the $\mathrm{H}^{-}$-source.

The radial density profile of the extracted beam can be measured by a CCD-Camera using the incident light emitted by collisions of the beam ions with the residual gas atoms. The measurements can be performed at different residual gas pressures.

\section{REFERENCE MEASUREMENTS WITHOUT MAG. DIPOL FIELDS}

Magnetic filter field are used near the extraction system of the ion source to generate negative ions. These fields influence the beam extraction and can be a source of emittance growth. Additionally they influence the current density delivered by the source. On the other hand theory of ion extraction shows that the extracted emittance for a given extraction system varies with the extracted beam current [9]. To distinguish these two effects on the beam emittance it was necessary to make the beam emittance insensitive to current variation by optimisation of the triode extraction system for wide range of current fluctuation [6]. This was done by carefully designing the extractor geometry by the use of numerical simulations[10].

To proof the results of the simulation and to have reference data we measured the emittance as function of the ion current in absence of the magnetic dipole fields. This is only possible for positive ions, because filter fields are essential for generation of negative ions. Fig. 3 shows the measured function $\varepsilon=\mathrm{f}(\mathrm{I})$ of the extraction system without dipole fields in the range of interest for the measurements with dipole fields (see. Fig. 5, next chapter). The ion current was varied by increasing the arc current of our source. All other source parameter have been kept constant.

The emittance was measured $38 \mathrm{~cm}$ behind the outlet aperture. The generalised perveance in this measurement varied between $\mathrm{K}=0.0011$ and $\mathrm{K}=0.003$.

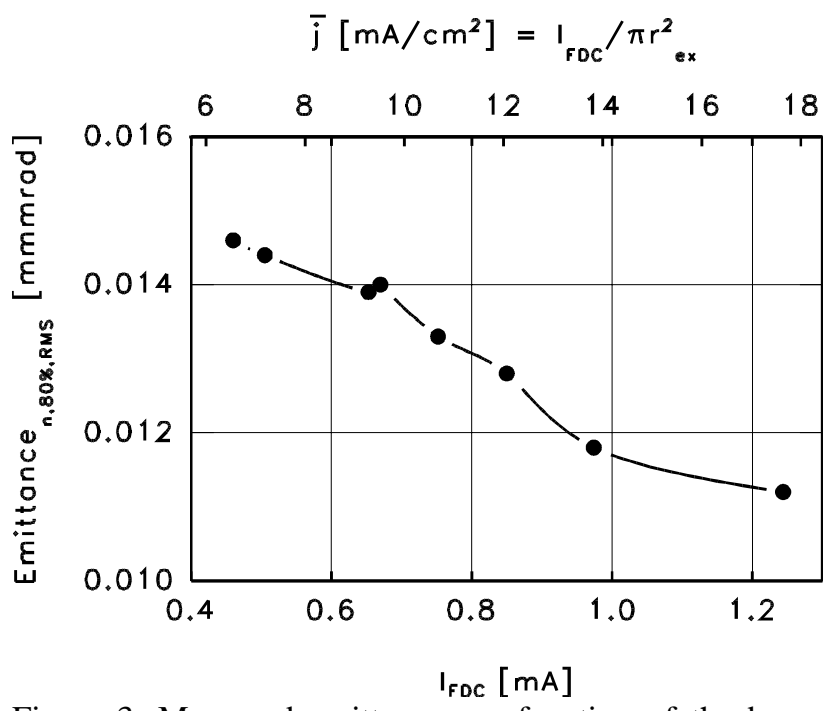

Figure 3: Measured emittance as function of the beam current for positive ions (extraction voltage $6 \mathrm{keV}$, radius of extraction hole $r_{e x}=1.5 \mathrm{~mm}$, see also fig. 4) .

The emittance decreases with increasing the extractable current. The absolute change of the emittance is app. $\mathcal{E}_{\mathrm{n}, 80 \%, \mathrm{RMS}}=0.003 \pi \mathrm{mmmrad}(40 \%)$ induced by a current variation of $300 \%$. Therefore an emittance growth larger than 0.003 can be interpreted as influence of the magnetic dipole fields.

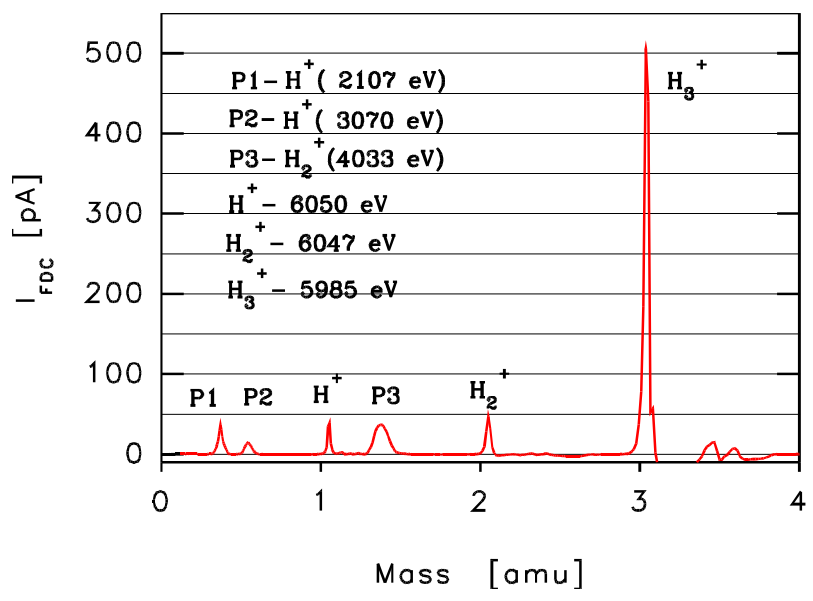

Figure 4: Result of the energy and mass spectrum of the extracted beam $(6 \mathrm{keV})$.

Fig4. shows the result of the energy and mass spectrum of the extracted beam. The main fraction of the positive ion peak was $\mathrm{H}_{3}^{+}$. Minor changes of the $\mathrm{H}^{+}$and $\mathrm{H}_{2}^{+}$fraction have been measured with filter fields. The ion energy of the peaks P1, P2 and P3 indicate that the ions are produced by interaction of $\mathrm{H}_{3}^{+}$with residual gas atoms in the extraction area. Their energy is determined by the momentum exchange at interaction. Further work to understand these results in more detail are planned. 


\section{$4 \quad \mathcal{E}$-MEASUREMENTS WITH MAGNETIC DIPOL FIELDS}

Second we measured the emittance as function of the fields of the bending and filter magnet (Fig.5). At the location of the plasma sheath the magnet is excited to about $20 \mathrm{G}$ per $1 \mathrm{~A}$. The negative current on the axis means an opposite polarity to the filter magnet. The current extracted from the source was in the range of Fig.3 (0.4 mA - $1.2 \mathrm{~mA})$. Thus all emittance growth larger than 0.003 can be interpreted as influence of the magnetic dipole fields. Without the help of the bending magnet some of the emittance was deflected partial out of the range of the emittance scanner. The grid is built by the measurements points, the coloured area corresponding to truncated emittances.

One can see an drastic emittance growth for exclusive use of the filter field. One can reduce this emittance growth (up to $500 \%$ ), which is an factor 10 higher than the influence of the extraction system (Fig.3) conteracting the influence of the filter by using the bending dipole magnet in opposite polarity. To understand the little influence of the bending magnet on beam emittance further experiments are planned. At present it seems helpful to reduce the emittance growth due to the necessary filter fields by use of the bending magnets. This measurements will be repeated with negative ions.

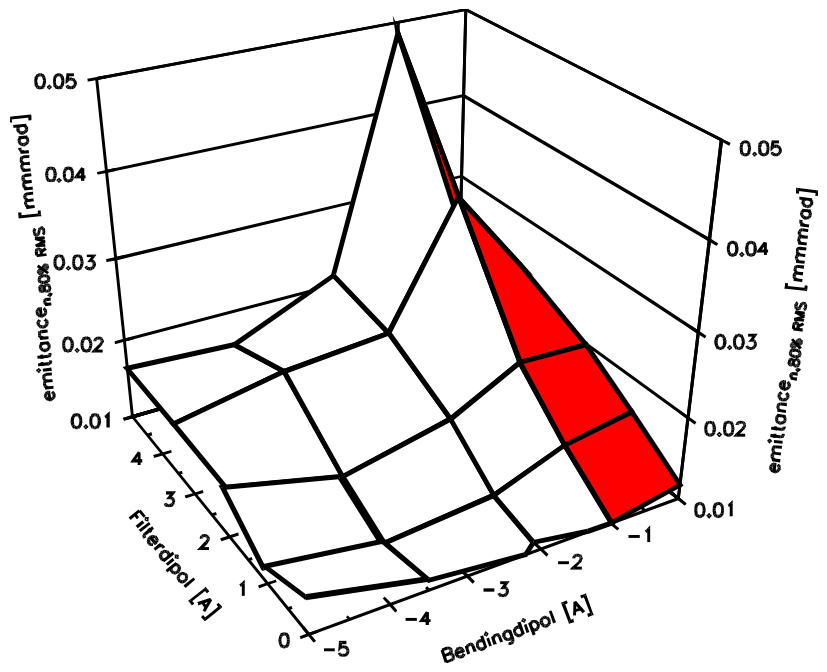

Figure 5: Influence of the dipole magnets on the measured emittance (negative current =opposite polarity)

\section{OUTLOOK}

After finishing the measurements on the test bench the source will be incorporated into the existing LEBT which is capable with the ESS scenario. The details of the beam line layout are shown in Fig. 6.

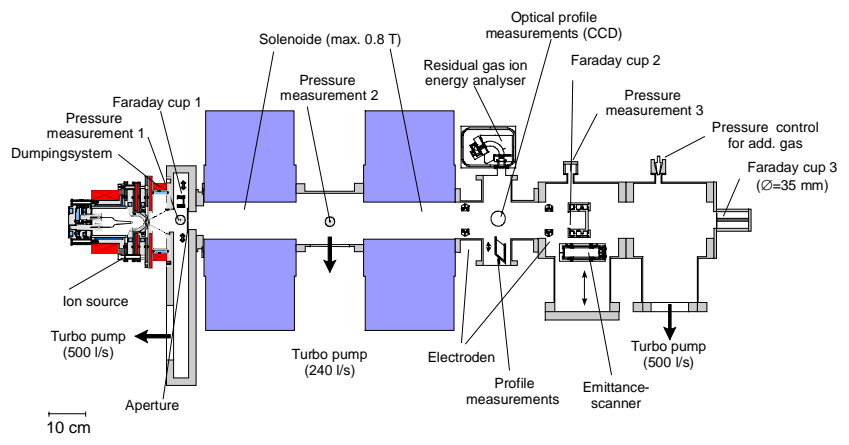

Figure 6: Schematic drawing of the experimental set up of the Frankfurt LEBT line.

First measurements to test the diagnostics (CCD, RGI) have already been performed using a beam of positive hydrogen ions with comparable beam parameters delivered by a different source. The extraction voltage was $6.6 \mathrm{kV}$, the ion current $3 \mathrm{~mA}$ measured after transport through the LEBT section $(\mathrm{K}=0.0042)$.

The experiments will start with a DC $\mathrm{H}^{-}$beam to study the influence of the external parameters (filter fields, solenoids, residual gas pressure, voltage on decompensation electrodes, source noise) on emittance and transmission. For a next step the set up is already prepared for pulsed mode operation.

\section{REFERENCES}

[1] H. Klein et. al., "The ESS Technical Study", ESS-9653-M, November 1996

[2] B.R. Appleton, J.B. Ball and J.R. Alonso , "The National Spallation Neutron Source", EPAC'96, Sitges, June 1996. p. 575

[3] J. W. Staples et al. , "All Electrostatic Split LEBT Test Results", LINAC'96, Geneva, August 1996. p. 157

[4] J. Pozimski, P.Groß, R. Dölling, K. Reidelbach and H.Klein, "LEBT-Design Studies for ESS", Proc. of ESS-PM4, October 1995, Weinfelden, PSI-Proc. 9502

[5] Ehlers, K. N. Leung et. al., "Increasing the efficiency of a multicusp ion source", Rev. Sci. Instr. 53(2), 1982, p. 1429

[6] A. Lakatos, J. Pozimski, A. Jakob, K. Klein, Proc. of EPAC 98, Stockholm, June 22-26, 1998, p.1400

[7] A. Lakatos, J. Pozimski, A. Jakob, K. Klein, Proc. of LINAC 98, Chicago, August 23-28, 1998, TU4056

[8] Ehlers, K. N. Leung et. al., "Effect of a magnetic filter on hydrogen ion species in a multicusp ion source", Rev. Sci. Instr. 52(2), 1981, p. 1452

[9] R. G. Wilson, G. R. Brewer, Ion Beams, John Wiley \& Sons, New York, London, Sydney, Toronto(1973)

[10] R. Becker, W. B.Herrmansfeldt, Proc. of the $4^{\text {th }}$ International Conference on Ion Source, Bensheim, Germany (1991) 